\title{
Overtube-assisted placement of a metal stent into the bile duct of a patient with surgically altered upper-gastrointestinal anatomy during double-balloon enteroscopy-assisted ERCP
}
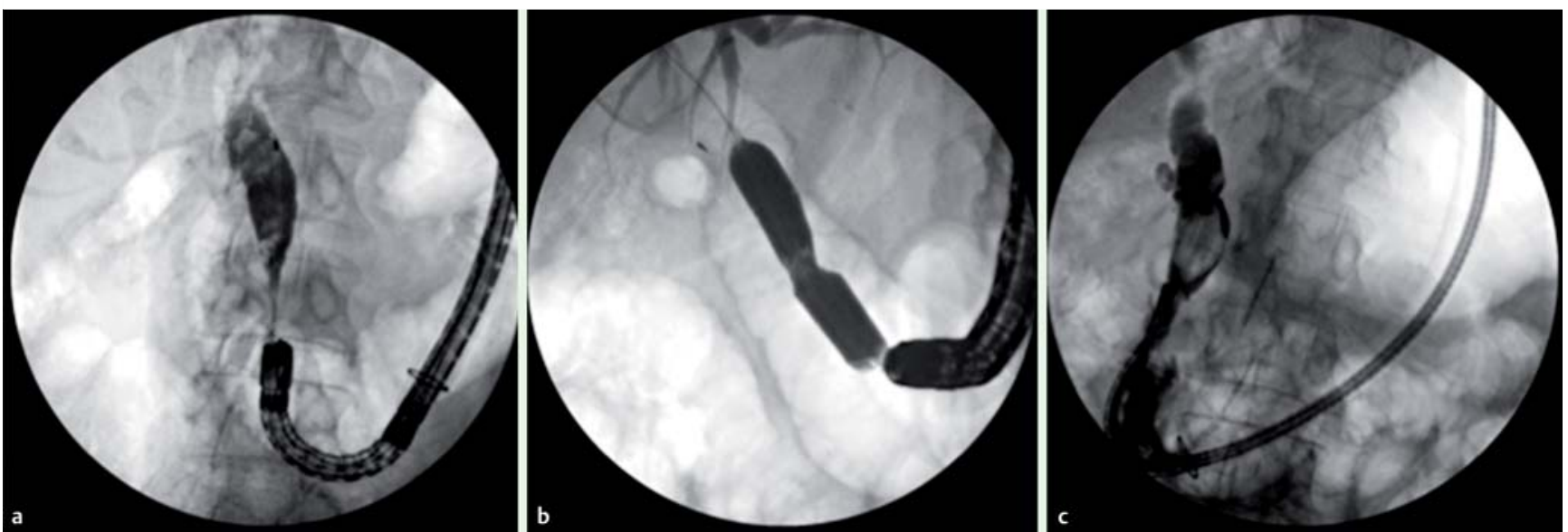

Fig. 1 Fluoroscopic images during double-balloon enteroscopy (DBE)-assisted endoscopic retrograde cholangiopancreatography (ERCP) in an 87-year-old woman with a history of a Billroth II reconstruction and complex choledocholithiasis showing: a multiple bile duct stones in the distally stenosed bile duct; b balloon sphincteroplasty being performed; c peroral direct cholangioscopic electrohydraulic lithotripsy being performed via an ultraslim gastroscope.
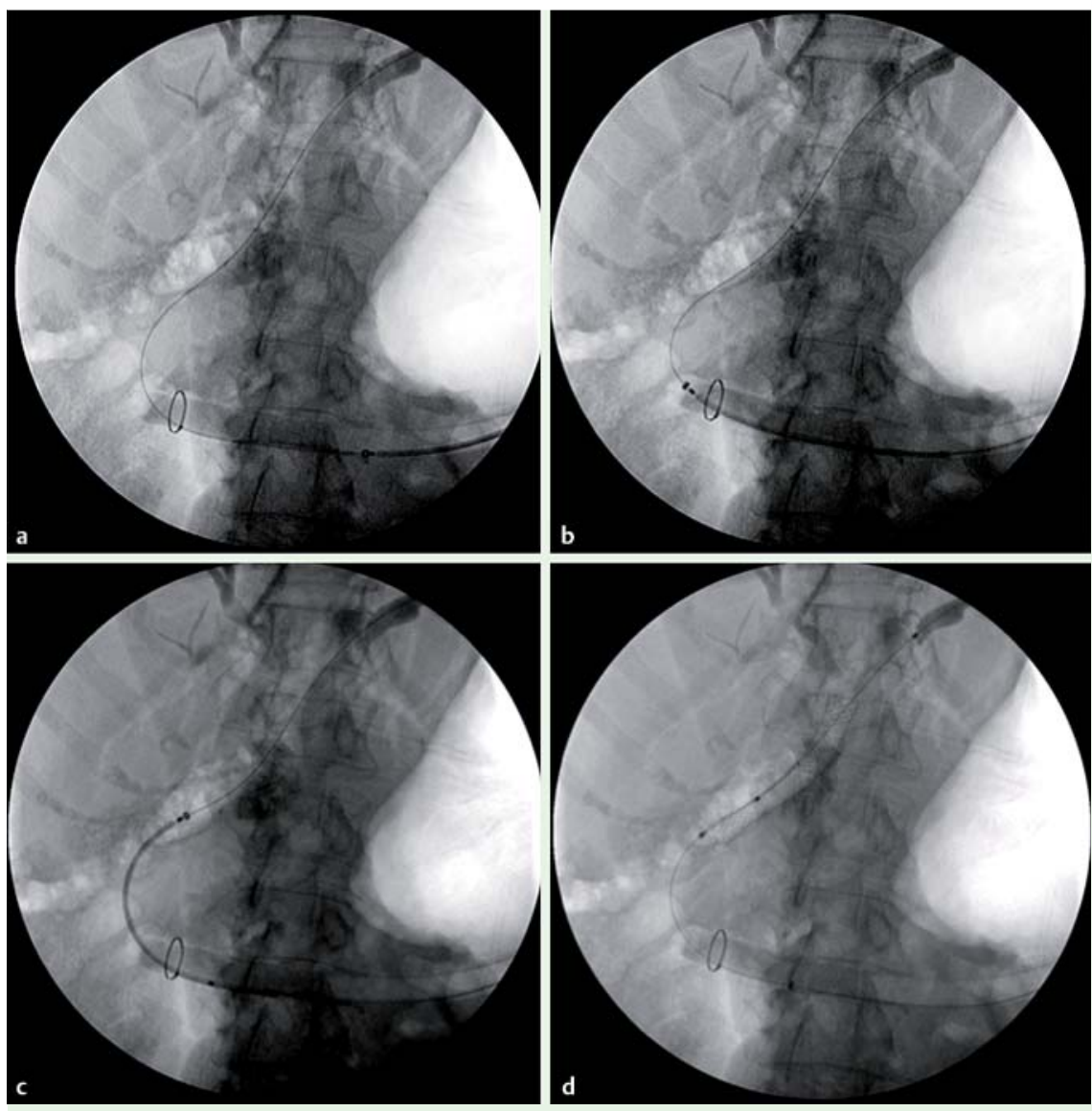

Fig. 2 Fluoroscopic images during double-balloon enteroscopy (DBE)-assisted endoscopic retrograde cholangiopancreatography (ERCP) showing: a the overtube left in place and the biliary guidewire deep in the biliary system after the scope had been removed; $\mathbf{b}$ a fully covered self-expanding metal stent (SEMS), which had been inserted using the overtube as a "large-diameter working channel", in position over the guidewire; $\mathbf{c}$ the SEMS following release; $\mathbf{d}$ the ring of the overtube, which was used to help estimate the location of the ampulla during fluoroscopy.

We here report on a novel approach to deliver a fully covered self-expanding metal stent (SEMS) into the bile duct during double-balloon enteroscopy (DBE)-assisted endoscopic retrograde cholangiopancreatography (ERCP) using the overtube as an "accessory" or working channel. An 87-year-old woman with a history of a Billroth II reconstruction many years previously and complex choledocholithiasis had previously undergone several ERCP attempts via both a colonoscope and a double-balloon enteroscope to clear the bile duct, which had been found to be dilated, tortuous, and distally stenotic (๑ Fig.1a).

A further DBE-ERCP (DBE-EN-450T5; Fujifilm, Saitama, Japan) was performed. During this procedure, a sphincteroplasty was performed using a $15-\mathrm{mm}$ controlled radial expansion (CRE) balloon (Boston Scientific; Natick, Massachusetts, USA; - Fig.1b). An electrohydraulic lithotriptor was then successfully delivered through an ultraslim gastroscope (GIF180; Olympus, USA), which had been advanced into the bile duct through an overtube ( $\bullet$ Fig. 1 c). After electrohydraulic lithotripsy (EHL) had been successfully performed, large amounts of sludge and stones were removed. Nevertheless, complete clearance of the stones was still not possible. 

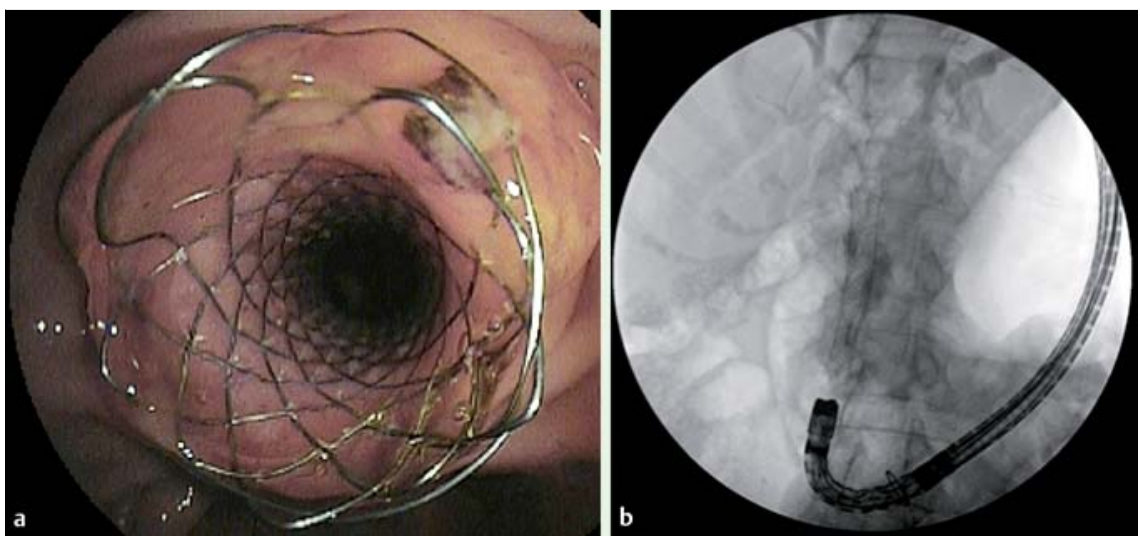

Fig. 3 Cholangiography was used to confirm the correct positioning of the stent: a endoscopic view; b fluoroscopic view.

The decision was therefore made to place a $10-\mathrm{mm}$ diameter, $60-\mathrm{mm}$ long, fully covered SEMS (Boston Scientific). The diameter of the stent delivery system was too large for the working channel of either an enteroscope or gastroscope. Therefore, the scope was removed, leaving the overtube in place and the biliary guidewire deep in the biliary system ( $\bullet$ Fig. 2a). A stent was then placed over the guidewire, with the overtube serving as a "large-diameter working channel" ( Fig. 2b). The stent was advanced and released under constant fluoroscopic guidance ( Fig. 2 c, d).

After the guidewire and delivery system had been removed, the enteroscope was passed through the overtube, which had been left in place, and the stent was directly visualized. It was confirmed to be in the correct position and an outflow of sludge, bile, and contrast medium was seen ( $\bullet$ Fig. 3).

Overtubes have previously been used to deliver SEMS through difficult strictures in the small intestine and colon [1,2]; however, as far as we are aware, there are no previous reports describing the placement of a SEMS into the bile duct using the overtube technique. The working channel of enteroscopes used for both DBE and single-balloon enteroscopy (SBE) are too small to permit the passage of larger accessories and to facilitate the deployment of larger diameter stents [3, 4].

In this case, fortunately, we were able to successfully keep the overtube stationary and close to the papilla in the afferent limb, where it could be used as a large accessory channel. The ring of the overtube allowed the precise position of the distal end of the overtube to be seen on fluoroscopy and the flexibility of the DBE overtube allowed the stent delivery system to be passed smoothly into the required position. We believe this technique is useful, and should be added to the therapeutic arsenal of the biliary endoscopist.

\section{Endoscopy_UCTN_Code_TTT_1AR_2AK}

Competing interests: None

\section{Matthew Skinner, Juan Pablo Gutierrez, C. Mel Wilcox, Klaus Mönkemüller}

Basil I. Hirschowitz Endoscopic Center of Excellence, Division of Gastroenterology and Hepatology, University of Alabama at Birmingham, USA

\section{References}

1 Samalin E, Assenat E, Bauret $P$ et al. Self-expandable metal stents placed with overtube for the treatment of malignant obstruction of the gastrointestinal tract in 33 consecutive patients. Endoscopy 2007; 39 (Suppl. 01): E101

2 Ross AS, Semrad C, Waxman I et al. Enteral stent placement by double balloon enteroscopy for palliation of malignant small bowel obstruction. Gastrointest Endosc 2006; 64: 835-837

3 Dellon ES, Kohn GP, Morgan DR et al. Endoscopic retrograde cholangiopancreatography with single-balloon enteroscopy is feasible in patients with a prior Roux-en-Y anastomosis. Dig Dis Sci 2009; 4: $1798-$ 1803

4 Neumann H, Fry LC, Meyer F et al. Endoscopic retrograde cholangiopancreatography using the single balloon enteroscope technique in patients with Roux-en-Y anastomosis. Digestion 2009; 80: $52-57$

\section{Bibliography}

Dol http://dx.doi.org/

10.1055/s-0033-1358806

Endoscopy 2013; 45: E418-E419

(c) Georg Thieme Verlag KG

Stuttgart · New York

ISSN 0013-726X

\section{Corresponding author}

\section{Klaus Mönkemüller, MD, PhD}

Division of Gastroenterology and Hepatology Basil Hirschowitz Endoscopic Center of Excellence Endoscopy Unit, JT 664

619 19th Street S

Birmingham

Alabama 35249

USA

klaus1@uab.edu 